\section{Comparison of Clinical Features of Pediatric Respiratory Syncytial Virus and Human Metapneumovirus Infections}

To the Editor-Respiratory syncytial virus (RSV) has long been known to be an important cause of morbidity and mortality in the young pediatric age group. The more recently identified human metapneumovirus (hMPV) is now increasingly recognized as a cause of acute respiratory tract disease in all age groups, ${ }^{1,2}$ with symptomatic disease most often seen in young children, especially those less than 1 year old. ${ }^{3,4}$ Infections due to hMPV are an important cause of pediatric hospitalizations, ${ }^{5,6}$ but to our knowledge the spectrum of clinical manifestations has not been fully defined.

In this study, we determined the local prevalence of hMPV infection and RSV infection among children aged 0-60 months during the winter seasons from November 2004 through April 2006 and compared the clinical manifestations of the 2 infections in a subset of the study population. We also examined the influence of comorbidities on the clinical course of the diseases.

Studies were performed under a protocol approved by the institutional review board of North Shore University Hospital. Discarded respiratory tract samples (nasopharyngeal aspirates, nasopharyngeal washes, nasopharyngeal swabs in viral transport media [Universal Transport Medium, Diagnostic Hybrids], transtracheal aspirates, and sputum), collected during the period from November 2004 through April 2006 and stored at $-70^{\circ} \mathrm{C}$, were obtained from 653 children ( 433 children aged 0-24 months and 220 children aged 25-60 months) who were symptomatic for a respiratory disease. Samples were submitted to the North Shore University Hospital Clinical Virology Laboratory, Manhasset, New York, for routine respiratory virus testing by means of direct immunofluorescence for influenza A, influenza B, parainfluenza 1, 2, and 3, adenovirus, and RSV and by means of R-Mix rapid cell culture (Diagnostic Hybrids). Total nucleic acids were extracted from a $200-\mu \mathrm{L}$ specimen aliquot by using NucliSENS magnetic silica extraction reagents (bioMérieux) and the NucliSENS miniMAG or NucliSENS easyMAG extraction platforms (bioMérieux). Molecular detection of RSV RNA and hMPV RNA was performed by using NucliSENS analyte-specific reagents (bioMerieux) according to in-house validated protocols and published studies. ${ }^{7.8}$

Standardized case report forms were completed by means of retrospective review of the medical records of a subset of patients who tested positive for RSV, hMPV, or both and for whom medical records were available. Patient demographics, clinical findings, comorbidities, and radiologic results were recorded. Fever was defined as any temperature greater than $38^{\circ} \mathrm{C}$. Comorbidity was defined as any other diagnosis that was present at the time of the evaluation, for example, a history of substantial prematurity, reactive airway disease,

TA BLE 1. Comparison of Patient Characteristics and Clinical Features in Human Metapneumovirus (hMPV) and Respiratory Syncytial Virus (RSV) Infections

\begin{tabular}{lccc}
\hline & $\begin{array}{c}\text { hMPV-infected } \\
\text { patients } \\
(n=26)\end{array}$ & $\begin{array}{c}\text { RSV-infected } \\
\text { patients } \\
(n=35)\end{array}$ & $P$ \\
Characteristic & & & .916 \\
Age & 17 & 23 & $\ldots$ \\
$0-24$ months & 9 & 12 & $\ldots$ \\
$25-60$ months & 19 & 22 & .400 \\
Comorbidities & 8 & 10 & $>.99$ \\
Upper respiratory tract infection & 10 & 19 & .301 \\
Lower respiratory tract infection & 5 & 4 & .477 \\
Mechanical ventilation & 13 & 1 & $<.001$ \\
Fever & 16 & 24 & .568 \\
Cough & 4 & 9 & .367 \\
Vomiting & 1 & 9 & .034 \\
Diarrhea & 5 & 0 & .011 \\
Rash & 2 & 15 & .003 \\
Rhinorrhea & 4 & 11 & .230 \\
Retractions & 6 & 8 & .984 \\
Wheeze & 6 & 12 & .343 \\
Crackles & 9 & 6 & $>.99$ \\
Pneumonia & 12 & 8 & .055 \\
Chest radiography performed & 19 & 22 & .481 \\
Antibiotics prescribed & 1.3 & 3.9 & .878 \\
Duration of pediatric intensive care unit stay, days, mean & 3.6 & .855 \\
Duration of hospital stay, days, mean & & 3.5 &
\end{tabular}

NOTE. Data are no. (\%) of patients, unless otherwise indicated. 
chronic lung disease, congenital heart disease, muscular disease, or malignancy.

Statistical analysis was performed by means of SAS statistical analysis software, version 9.1.3 (SAS Institute). The $\chi^{2}$ test or Fisher exact test was used to compare parameters. For nonparametric comparisons, that is, length of pediatric intensive care unit stay and length of hospital stay, the Wilcoxon rank sum test was used. A $P$ value of less than .05 was considered to indicate a statistically significant difference.

We collected 653 unique patient specimens during the study period. A combination of all test methods identified 143 specimens (22\%) as RSV positive (113 [79\%] of 143 RSVpositive specimens were from children aged 0-24 months, and $30[21 \%]$ of 143 RSV-positive specimens were from children aged 24-60 months) and 35 specimens (5\%) as hMPV positive ( 25 [71\%] of $35 \mathrm{hMPV}$-positive specimens were from children aged 0-24 months, and 10 [29\%] of 35 hMPVpositive specimens were from children aged 24-60 months). RSV infections peaked in January, and hMPV infections peaked in March and April. Hospitalization was required for $35(24 \%)$ of 143 RSV-infected children, and $4(11 \%)$ of the 35 hospitalized children were admitted to the pediatric intensive care unit. A significantly higher proportion of hMPVinfected children, $20(57 \%)$ of 35 , were hospitalized $(P<$ .01 ), and $4(20 \%)$ of the 20 hospitalized children were admitted to the pediatric intensive care unit.

The clinical comparison of 61 children with RSV infection and/or hMPV infection is presented in the Table. Overall, the clinical manifestations of hMPV infection were similar to those of RSV infection, as in other studies. ${ }^{6,9,10}$ However, children with hMPV infection were significantly more likely to present with a fever or with a rash, whereas RSV-infected children were significantly more likely to have diarrhea or rhinorrhea. Rashes were not uniformly described in all medical records. Three children had a nonspecified type of rash on the face, neck, or palms, and 2 children had a maculopapular rash with no location noted. A rash was also described in $16.7 \%$ of hMPV-infected patients in a Canadian study ${ }^{9}$ and in $12.9 \%$ of hMPV-infected children in a Hong Kong study; in the Hong Kong study, the duration of the rash was a few hours to 1 day." RSV-infected and hMPV-infected patients were equally likely to have an underlying comorbidity. Coinfection with hMPV and RSV was extremely uncommon, and there was no evidence that respiratory disease was more severe in coinfected children.

The higher rate of hospitalization in hMPV-infected children may have been due to the lack of a specific diagnosis at the time of presentation, because the routine direct immunofluorescence and R-mix culture did not screen for hMPV (the results of the hMPV molecular testing were not available to the clinician). In the future, the availability of rapid testing for hMPV could reduce unnecessary hospitalization, as well as inappropriate antibiotic use. Additional studies, to evaluate further the clinical and economic effect of rapid diagnosis by means of molecular testing, would be valuable.
There were some limitations to our study. The relatively small number of medical records available for review may have skewed results toward patients who were hospitalized, because those charts were more readily available. In addition, the data that we obtained retrospectively are limited by the subjectivity inherent in information recorded by physicians.

\section{ACKNOWLEDGMENTS}

Potential conflicts of interest. C.C.G. reports that she has received grant support from bioMérieux. All authors report no conflicts of interest relevant to this article.

\section{Beneri, DO; C. C. Ginocchio, PhD; R. Manji, BS; S. Sood, MD}

From Stony Brook University Medical Center, Stony Brook (C.B.), North Shore University Hospital, Manhasset (C.C.G., S.S.), and North Shore-Long Island Jewish Health System Laboratories, Lake Success (C.C.G., R.M.), New York.

Address reprint requests to Christy Beneri, DO, SUNY Stony Brook, HSC T11 Room 030, Stony Brook, NY 11794 (cbeneri@notes.cc.sunysb.edu). Infect Control Hosp Epidemiol 2009; 30:1240-1241

(c) 2009 by The Society for Healthcare Epidemiology of America. All rights reserved. 0899-823X/2009/3012-0022\$15.00. DOI: $10.1086 / 648665$

\section{REFERENCES}

1. Boivin G, De Serres G, Hamelin M, et al. An outbreak of severe respiratory tract infection due to human metapneumovirus in a long-term care facility. Clin Infect Dis 2007; 44:1152-1158.

2. McAdam AJ, Hasenbein ME, Feldman HA, et al. Human metapneumovirus in children tested at a tertiary-care hospital. I Infect Dis 2004; 190:20-26.

3. Esper F, Martinello RA, Boucher D, et al. A 1-year experience with human metapneumovirus in children aged $<5$ years. J Infect Dis 2004; 189: 1388-1396.

4. Van Den Hoogen BG, Osterhaus ME, Foucher RAM. Clinical impact and diagnosis of human metapneumovirus infection. Pediatr Infect Dis J 2004; 23(Suppl 1):S25-S32.

5. Al-Sonbali N, Hart $\mathrm{CH}, \mathrm{Al}$-Aeryani A, et al. Respiratory syncytial virus and human metapneumovirus in children with acute respiratory infections in Yemen. Pediatr Infect Dis J 2005; 24:734-736.

6. Wilkesmann A, Schildgen O, Eis-Hübinger AM. Human metapneumovirus infections cause similar symptoms and clinical severity as respiratory syncytial virus infections. Eur J Pediatr 2006; 165:467-475.

7. Moore C, Valappil M, Corden S, Westmoreland D. Enhanced clinical utility of the NucliSens EasyQ RSV A+B assay for rapid detection of respiratory syncytial virus in clinical samples. Eur J Clin Microbiol Infect Dis 2006; 25:167-174.

8. Ginocchio CC, Manji M, Zhang F. Clinical evaluation of NucliSENS magnetic extraction and NucliSENS analytic specific reagents for the real time detection of human metapneumovirus (hMPV) in pediatric respiratory specimens. J Clin Microbiol 2008; 46:1274-1278.

9. Hamelin M, Abed $Y$, Boivin G. Human metapneumovirus: a new player among respiratory viruses. Clin Infect Dis 2004; 38:983-990.

10. Wolf DG, Greenberg D, Kalkstein D, et al. Comparison of human metapneumovirus, respiratory syncytial virus, and influenza $A$ virus lower respiratory tract infections in hospitalized young children. Pediatr Infect Dis J 2006; 25:320-324.

11. Peiris JSM, Tang W, Chan $\mathrm{K}$, et al. Children with respiratory disease associated with metapneumovirus in Hong Kong. Emerg Infect Dis 2003; 9:628-633. 